\title{
A NOTE ON THE PERIODIC ORBITS OF A SELF EXCITED RIGID BODY
}

\author{
JOSE M. FERRÁNDIZ ${ }^{1}$, JUAN L. G. GUIRAO ${ }^{2}$ AND JUAN A. VERA ${ }^{3}$
}

Abstract. The aim of the present paper is to study the periodic orbits of a perturbed self excited rigid body with a fixed point. For studying these periodic orbits we shall use averaging theory of first order.

\section{Introduction AND STATEMENT OF THE MAIN RESUlts}

The equations of the motion of a rigid body with moments of inertia $A, B, C$ $(A \leq B \leq C)$ under a external torque $\mathcal{M}=\left(M_{1}, M_{2}, M_{3}\right)$ are

$$
\begin{aligned}
\frac{d \pi_{1}}{d t} & =\left(\frac{B-C}{B C}\right) \pi_{2} \pi_{3}+M_{1}, \\
\frac{d \pi_{2}}{d t} & =\left(\frac{C-A}{A C}\right) \pi_{1} \pi_{3}+M_{2}, \\
\frac{d \pi_{3}}{d t} & =\left(\frac{A-B}{A B}\right) \pi_{1} \pi_{2}+M_{3},
\end{aligned}
$$

where $\Pi=\left(\pi_{1}, \pi_{2}, \pi_{3}\right)$ is the total angular momentum of the rigid body and $M_{i}$ are smooth functions depending on the variables $\pi_{1}, \pi_{2}, \pi_{3}$. This rigid body is usually called at the literature self-excited rigid body, see for more information on this mechanical system Leimanis [9] or Deprit et alt. [2] In the following, we assume that we work with a rigid body quasi-axisymmetric under small torques, i.e. $B=A+\varepsilon$, and the functions $M_{i}=\varepsilon f_{i}\left(\pi_{1}, \pi_{2}, \pi_{3}\right)$ with $\varepsilon$ a small real parameter. Under these assumptions, very usual in many practical applications, the equations of motion (1) are reduced to

$$
\begin{aligned}
\frac{d \pi_{1}}{d t} & =-\left(\frac{C-A-\varepsilon}{(A+\varepsilon) C}\right) \pi_{2} \pi_{3}+\varepsilon f_{1}\left(\pi_{1}, \pi_{2}, \pi_{3}\right), \\
\frac{d \pi_{2}}{d t} & =\left(\frac{C-A}{A C}\right) \pi_{1} \pi_{3}+\varepsilon f_{2}\left(\pi_{1}, \pi_{2}, \pi_{3}\right), \\
\frac{d \pi_{3}}{d t} & =-\left(\frac{\varepsilon}{A(A+\varepsilon)}\right) \pi_{1} \pi_{2}+\varepsilon f_{3}\left(\pi_{1}, \pi_{2}, \pi_{3}\right),
\end{aligned}
$$

Key words and phrases. Rigid body with a fixed point, periodic orbits, averaging theory. 2010 Mathematics Subject Classification. Primary: 70E17, 70E20, 70E40. Secondary: $37 \mathrm{C} 27$.

Corresponding author affiliation:Departamento de Matemática Aplicada y Estadística. Universidad Politécnica de Cartagena, Hospital de Marina, 30203-Cartagena, Región de Murcia, Spain., e-mail:juan.garcia@upct.es . 
The objective of this work is, by means of the Averaging Theory (see Theorem 4 of the Appendix) to provide a system of nonlinear equations whose simple zeros provide periodic solutions of (2). This idea has been used in previous works as an integrability criterium and for obtaining approximation solutions to real problems, see for instance $[3,4,5,6]$. In order to present our results we need some preliminary definitions and notation.

We define the following functions in the open domain $\Omega=\left\{(R, Z) \in \mathbb{R}^{2} /\right.$ $0<R<+\infty, 0<Z<+\infty\}$ given by, $\mathcal{F}_{1}(R, Z)$ as

$$
\begin{aligned}
& \frac{1}{\pi Z} \int_{0}^{2 \pi}\left(\cos \theta f_{1}\left(\frac{R \cos \theta}{2 \alpha}, \frac{R \sin \theta}{2 \alpha}, Z\right)+\sin \theta f_{2}\left(\frac{R \cos \theta}{2 \alpha}, \frac{R \sin \theta}{2 \alpha}, Z\right)\right) d \theta \\
& \text { and } \mathcal{F}_{2}(R, Z)=\frac{2 \alpha}{\pi Z} \int_{0}^{2 \pi} f_{3}\left(\frac{R \cos \theta}{2 \alpha}, \frac{R \sin \theta}{2 \alpha}, Z\right) d \theta,
\end{aligned}
$$

with $\alpha=\frac{C-A}{C A}$. A zero $\left(R_{0}^{*}, Z_{0}^{*}\right)$ of the nonlinear system

$$
\mathcal{F}_{1}(R, Z)=0, \quad \mathcal{F}_{2}(R, Z)=0,
$$

such that

$$
\operatorname{det}\left(\left.\frac{\partial\left(\mathcal{F}_{1}, \mathcal{F}_{2}\right)}{\partial(R, Z)}\right|_{(R, Z)=\left(R_{0}^{*}, Z_{0}^{*}\right)}\right) \neq 0,
$$

is called a simple zero of system (4).

Our main results on the periodic solutions of the self-excited rigid body (2) are the following.

Theorem 1. Then for $\varepsilon \neq 0$ sufficiently small and for every simple zero $\left(R_{0}^{*}, Z_{0}^{*}\right) \neq(0,0)$ of the nonlinear system (4), then the self-excited rigid body (2) has a periodic solution $\left(\pi_{1}(t, \varepsilon), \pi_{2}(t, \varepsilon), \pi_{3}(t, \varepsilon)\right)$ tending when $\varepsilon \rightarrow 0$ to $\left(\frac{R_{0}^{*}}{2 \alpha}, 0, Z_{0}^{*}\right)$.

Theorem 1 is proved in section 2. Its proof is based in the averaging theory for computing periodic solutions, see the appendix.

We provide two applications of Theorem 1 in the following two corollaries, which will be proved in section 3 , and example of perturbed maps where Theorem 1 not provides information, see section?? for details.

Corollary 2. If $f_{1}\left(\pi_{1}, \pi_{2}, \pi_{3}\right)=-\pi_{3}^{2} \pi_{1}, f_{2}\left(\pi_{1}, \pi_{2}, \pi_{3}\right)=\frac{2}{3}\left(\frac{\pi_{3}^{3} \pi_{2}}{2}+\pi_{3} \pi_{2}\right)$ and $f_{3}\left(\pi_{1}, \pi_{2}, \pi_{3}\right)=\pi_{3}^{2}-2 \pi_{3}^{4} \pi_{1}^{2}$ then the differential equation (2) for $\varepsilon \neq 0$ sufficiently small has two periodic solutions $\left(\pi_{1}(t, \varepsilon), \pi_{2}(t, \varepsilon), \pi_{3}(t, \varepsilon)\right)$ tending when $\varepsilon \rightarrow 0$ to the following initial conditions

$$
(1,0,1) \text { and }\left(X_{0}^{*}, Y_{0}^{*}\right)=\left(\frac{1}{2}, 0,2\right)
$$


respectively.

Corollary 3. If $f_{1}\left(\pi_{1}, \pi_{2}, \pi_{3}\right)=-\alpha\left(\pi_{1}+\pi_{2}\right), f_{2}\left(\pi_{1}, \pi_{2}, \pi_{3}\right)=\alpha^{3} \pi_{2}^{3}$ and $f_{3}\left(\pi_{1}, \pi_{2}, \pi_{3}\right)=1-\alpha^{2} \pi_{1}^{2} \pi_{3}$ then the differential equation (2) for $\varepsilon \neq 0$ sufficiently small has one periodic solution $\left(\pi_{1}(t, \varepsilon), \pi_{2}(t, \varepsilon), \pi_{3}(t, \varepsilon)\right)$ tending when $\varepsilon \rightarrow 0$ to the following initial condition $\left(\frac{2 \sqrt{3}}{3 \alpha}, 0, \frac{3}{2}\right)$.

\section{Proof of the Theorem 1}

Proof of the Theorem 1. Introducing the change of variables

$$
\pi_{1}=\frac{1}{2 \alpha} R \cos \theta, \quad \pi_{2}=\frac{1}{2 \alpha} R \sin \theta, \quad \pi_{3}=Z,
$$

in the system (2) by means of the following relations

$$
\frac{d R}{d t}=\frac{\pi_{1} \frac{d \pi_{1}}{d t}+\pi_{2} \frac{d \pi_{2}}{d t}}{R}, \quad \frac{d \theta}{d t}=\frac{\pi_{1} \frac{d \pi_{2}}{d t}-\pi_{2} \frac{d \pi_{1}}{d t}}{R^{2}}, \quad \frac{d Z}{d t}=\frac{d \pi_{3}}{d t},
$$

we obtain, to first order in the small parameter $\varepsilon$, the following system of differential equations

$$
\begin{aligned}
& \frac{d R}{d t}=\varepsilon F_{1}(R, \theta, Z)+O\left(\varepsilon^{2}\right), \\
& \frac{d \theta}{d t}=\frac{Z}{4 \alpha}+O(\varepsilon), \\
& \frac{d Z}{d t}=\varepsilon F_{2}(R, \theta, Z)+O\left(\varepsilon^{2}\right),
\end{aligned}
$$

with

$$
\begin{gathered}
F_{1}(R, \theta, Z)=\frac{\Delta}{4 \alpha^{2} C^{2}} \\
F_{2}(R, \theta, Z)=f_{3}\left(\frac{R \cos \theta}{2 \alpha}, \frac{R \sin \theta}{2 \alpha}, Z\right)-\frac{R^{2}(1+\alpha C)^{2} \sin 2 \theta}{8 \alpha^{2} C} .
\end{gathered}
$$

where $\Delta$ is equal to $2 \alpha C^{2} \cos \theta f_{1}\left(\frac{R \cos \theta}{2 \alpha}, \frac{R \sin \theta}{2 \alpha}, Z\right)+2 \alpha C^{2} \sin \theta$ $f_{2}\left(\frac{R \cos \theta}{2 \alpha}, \frac{R \sin \theta}{2 \alpha}, Z\right)+R Z(1+\alpha C)^{2} \cos \theta$.

Using the equation $\frac{d \theta}{d t}=\frac{Z}{4 \alpha}+O(\varepsilon)$ the system (5) is reduced to a planar system, $2 \pi$-periodic in the variable $\theta$ in the following form

$$
\begin{aligned}
& \frac{d R}{d \theta}=\varepsilon \frac{4 \alpha F_{1}(R, \theta, Z)}{Z}+O\left(\varepsilon^{2}\right), \\
& \frac{d Z}{d \theta}=\varepsilon \frac{4 \alpha F_{2}(R, \theta, Z)}{Z}+O\left(\varepsilon^{2}\right),
\end{aligned}
$$


in the set $\Omega_{1}=\left\{(R, \theta, Z) \in \mathbb{R}^{3} / 0<R<+\infty, \theta \in S_{1}, 0<Z<+\infty\right\}$. System (6) is in normal form for using, see the Appendix. Computing the averaged system we obtain

$$
\begin{aligned}
& \frac{d R}{d \theta}=\varepsilon \mathcal{F}_{1}(R, Z)+O\left(\varepsilon^{2}\right), \\
& \frac{d Z}{d \theta}=\varepsilon \mathcal{F}_{2}(R, Z)+O\left(\varepsilon^{2}\right),
\end{aligned}
$$

in the open set $\Omega=\left\{(R, Z) \in \mathbb{R}^{2} / 0<R<+\infty, 0<Z<+\infty\right\}$ with $\mathcal{F}_{1}(R, Z)$ and $\mathcal{F}_{2}(R, Z)$ given by $(3)$. Hence Theorem 1 is proved.

\section{Proof of the Corollary 2 AND 3}

Proof of the corollary 2. Under the assumptions of Corollary 2 the nonlinear system (4) becomes

$$
\begin{aligned}
& \mathcal{F}_{1}(R, Z)=\frac{R\left(2-3 Z+Z^{2}\right)}{6 \alpha}, \\
& \mathcal{F}_{2}(R, Z)=\frac{Z}{2}\left(8 \alpha-\frac{2 R^{2} Z^{2}}{\alpha}\right) .
\end{aligned}
$$

This system has the two real solutions

$$
\left(R_{0}^{*}, Z_{0}^{*}\right)=(2 \alpha, 1) \text { and }\left(R_{0}^{*}, Z_{0}^{*}\right)=(\alpha, 2) .
$$

Moreover

$$
\operatorname{det}\left(\left.\frac{\partial\left(\mathcal{F}_{1}, \mathcal{F}_{2}\right)}{\partial(R, Z)}\right|_{(R, Z)=(2 \alpha, 1)}\right)=-\frac{4}{3}
$$

and

$$
\operatorname{det}\left(\left.\frac{\partial\left(\mathcal{F}_{1}, \mathcal{F}_{2}\right)}{\partial(R, Z)}\right|_{(R, Z)=(\alpha, 2)}\right)=\frac{8}{3}
$$

check that these solutions are simple. So, by Theorem 1 we only have two periodic solutions of (2). This completes the proof of the corollary.

Proof of the corollary 3. Under the assumptions of Corollary 3 the nonlinear system (4) becomes

$$
\begin{aligned}
& \mathcal{F}_{1}(R, Z)=\frac{R\left(3 R^{2}-16\right)}{Z}, \\
& \mathcal{F}_{2}(R, Z)=\frac{\alpha\left(8-R^{2} Z\right)}{2 Z} .
\end{aligned}
$$

This system has the only solution

$$
\left(R_{0}^{*}, Z_{0}^{*}\right)=\left(\frac{4 \sqrt{3}}{3}, \frac{3}{2}\right) .
$$

Moreover

$$
\operatorname{det}\left(\left.\frac{\partial\left(\mathcal{F}_{1}, \mathcal{F}_{2}\right)}{\partial(R, Z)}\right|_{(R, Z)=\left(R_{0}^{*}, Z_{0}^{*}\right)}\right)=-\frac{32 \alpha}{27} \neq 0
$$


check that these solutions are simple. So, by Theorem 1 we only have one periodic solutions of (2). This completes the proof of the corollary.

\section{Appendix}

Now we shall present the basic results from averaging theory that we need for proving the results of this paper.

The next theorem provides a first order approximation for the periodic solutions of a periodic differential system, for the proof see [1] and [10].

Consider the differential equation

$$
\dot{x}=\varepsilon F_{1}(t, x)+\varepsilon^{2} R(t, x, \varepsilon), x(0)=x_{0}
$$

with $x \in D \subset \mathbb{R}^{n}, t \geq 0$. Moreover we assume that both $F_{1}(t, \mathrm{x})$ and $F_{2}(t, \mathrm{x})$ are $T$ periodic in $t$. Separately we consider in $D$ the averaged differential equation

$$
\dot{y}=\varepsilon f_{1}(y), y(0)=x_{0},
$$

where

$$
f_{1}(y)=\frac{1}{T} \int_{0}^{T} F_{1}(t, y) d t .
$$

Under certain conditions, equilibrium solutions of the averaged equation turn out to correspond with $T$-periodic solutions of equation (8).

Theorem 4. Consider the two initial value problems (7) and (8). Suppose:

(i) $F_{1}$, its Jacobian $\partial F_{1} / \partial x$, its Hessian $\partial^{2} F_{1} / \partial x^{2}, F_{2}$ and its Jacobian $\partial F_{2} / \partial x$ are defined, continuous and bounded by an independent constant $\varepsilon$ in $[0, \infty) \times D$ and $\varepsilon \in\left(0, \varepsilon_{0}\right]$.

(ii) $F_{1}$ and $F_{2}$ are $T$-periodic in $t$ ( $T$ independent of $\varepsilon$ ).

(iii) $y(t)$ belongs to $\Omega$ on the interval of time $[0,1 / \varepsilon]$.

Then the following statements hold.

(a) For $t \in[1, \varepsilon]$ we have that $x(t)-y(t)=O(\varepsilon)$, as $\varepsilon \rightarrow 0$.

(b) If $p$ is a singular point of the averaged equation (8) and

$$
\left.\operatorname{det}\left(\frac{\partial\left(f_{1}+\varepsilon f_{2}\right)}{\partial y}\right)\right|_{\mathrm{y}=p} \neq 0,
$$

then there exists a T-periodic solution $\varphi(t, \varepsilon)$ of equation (7) which is close to $p$ such that $\varphi(0, \varepsilon) \rightarrow p$ as $\varepsilon \rightarrow 0$.

\section{ACKNOWLEDGEMENTS}

The first and third authors was partially supported by MICINN/FEDER grant number MTM2011-22587. The second author was partially supported by AYA 2010 Ministerio de Ciencia e Innovación grant number 22039-C0201 and ACOMP Cosellería de Educación de la Generalitat Valenciana grant number 2012/128. 


\section{REFERENCES}

[1] A. Buica And J. LliBRe, Averaging methods for finding periodic orbits via Brouwer degree, Bulletin de Sciences Mathemàtiques 128 (2004), 7-22.

[2] A. Deprit and A. Elipe, Complete reduction of the Euler-Poinsot problem, J. Astron. Sci. 41 (1993), 603-628.

[3] J.M. Ferrándiz, J.F. Navarro, A. Escada and J. Getino, Precession of the non rigid earth: effect of the fluid outer core, The Astronomical Journal 128 (2004), 14071411.

[4] J. Getino And J.M. Ferrándiz, Forced nutations of a two-layer Earth model, Mon. Not. R. Astrom. Soc. 322 (2001), 785-799.

[5] J.L.G. Guirao, J. Llibre And J.A. Vera, Generalized van der Waals Hamiltonian: Periodic orbits and $C^{1}$ nonintegrability, Physical Review E 85 (2012), 036603.

[6] M. T. de Bustos, J. L. G. Guirao, J. A. Vera and J. Vigo-Aguiar, Periodic orbits and $\mathcal{C}^{1}$-integrability in the planar Stark-Zeeman problem, J. Math. Phys. 53, 082701 (2012).

[7] L. Jiménez-Lara and J. Llibre, Periodic orbits and non-Integrability of Henon-Heiles system, J. Physics A: Maths. Gen. 44 (2011), 205103-14 pp.

[8] L. Jiménez-Lara And J. Llibre, Periodic orbits and non-integrability of generalized classical Yang-Mills Hamiltonian system, J. Math. Phys., 52 (2011), 032901-9 pp.

[9] E. Leimanis, The general problem of the motion of coupled rigid bodies about a fixed point, Springer Verlag Berlin, 1965.

[10] J. LliBRE, Averaging theory and limit cycles for quadratic systems, Radovi Matematicki 11, (2002), 215-228.

1 Departamento de Matemática Aplicada. Universidad de Alicante, Escuela Politécnica Superior, Ap. De Correos 99, 03080-Alicante

E-mail address: jm.ferrandiz@ua.es

2 Departamento de Matemática Aplicada y Estadística. Universidad Politécnica de Cartagena, Hospital de Marina, 30203-Cartagena, Región de Murcia, Spain. -Corresponding Author-

E-mail address: juan.garcia@upct.es

3 Centro Universitario de la Defensa. Academia General del Aire. Universidad Politécnica de Cartagena, 30720-Santiago de la Ribera, Región de Murcia, Spain

E-mail address: juanantonio.vera@cud.upct.es 